\title{
Thyroid abnormalities in paediatric patients with vitiligo: retrospective study
}

\author{
Demet Kartal ${ }^{1}$, Murat Borlu1, Salih Levent Çınar ${ }^{1}$, Ayten Kesikoğlư ${ }^{1}$, Serap Utaş ${ }^{2}$ \\ ${ }^{1}$ Department of Dermatology and Venereology, Faculty of Medicine, Erciyes University, Kayseri, Turkey \\ ${ }^{2}$ Department of Dermatology and Venereology, Acıbadem Hospital, Istanbul, Turkey
}

Adv Dermatol Allergol 2016; XXXIII (3): 232-234

DOI: 10.5114/ada.2016.60617

\begin{abstract}
Introduction: The association between vitiligo and thyroid disease is not fully investigated especially in paediatric patients.

Aim: To determine the incidence of vitiligo and thyroid disorders in children. This is the first report from middle Anatolia and the second report from Turkey.

Material and methods: A retrospective chart review was performed to examine the presence of thyroid abnormalities in paediatric patients who had been admitted to the dermatology department with vitiligo.

Results: A total of 155 paediatric patients, including 80 (52\%) male and 75 (48\%) female patients were included. The mean age was 8.6 years. Non segmental vitiligo was the most common type of the disease in 140 (90\%) reviewed patients, while segmental vitiligo appeared only in 15 (10\%) patients. The mean onset of vitiligo was $5.6 \pm 0.9$ years. A family history of vitiligo was found in 14 (9\%) children. Thirty-four (22\%) patients had thyroid function tests and/ or thyroid autoantibody abnormality. All of these patients had non segmental vitiligo. It was statistically significant $(p<0.05)$ in types of vitiligo and thyroid disease parameters.

Conclusions: Our results show that it may be useful to screen thyroid in children with non segmental vitiligo.
\end{abstract}

Key words: vitiligo, thyroid abnormalities.

\section{Introduction}

Vitiligo is an acquired depigmentary disorder characterized by the loss of melanocytes from the epidermis. Clinically it is classified as segmental vitiligo (SV) and non-segmental vitiligo (NSV). The studies have shown that vitiligo patients often have melanocyte-specific antibodies [1] and circulating skin-homing melanocyte-specific cytotoxic T lymphocytes (CTLS) in their blood [2]. For NSV this autoimmunity against melanocytes is much more evident [3]. Thyroid disease seems to be the most common associated disorder in vitiligo patients. In adults its prevalence is $10 \%$, while the frequency in the general population is around $10 \%$. However in paediatric patients there are only a few reports about the incidence of thyroiditis [4-10].

\section{Aim}

The aim of our study was to determine the percentage of thyroid disease in paediatric patients with vitiligo.

\section{Material and methods}

An institutional review board-approved chart review of paediatric patients treated for vitiligo in the Erciyes University Medical Faculty, Dermatology Department Outpatient Clinic, from 2008-2013, was conducted. These charts were reviewed for characterization of vitiligo, age, age at onset, gender, personal and family history of thyroid disease. As a part of routine clinical evaluation in paediatric patients with vitiligo, we ordered thyroid function testing for thyroxine (T4), triiodothyronine (T3), thyrotropin (TSH), anti-thyroid peroxidase (anti-TPO) and anti-thyroglobulin (anti-Tg) antibodies. The patients with positive antibodies and/or abnormal thyroid function were consulted by a paediatric endocrinologist.

\section{Statistical analysis}

Statistical data were analyzed using the Fisher's exact test.

Address for correspondence: Demet Kartal MD, Dermatology and Venereology Department, Faculty of Medicine, Erciyes University, 38039 Kayseri, Turkey, phone: +90 3522076666-21916, fax: +90 352 4377615, mobile: 05333544101, e-mail: demetkartal@hotmail.com Received: 26.05.2015, accepted: 5.06.2015. 


\section{Results}

We reviewed the charts of 155 paediatric patients with vitiligo, 80 were boys and 75 were girls. The mean age of children was 8.6 years and the mean age of onset of the disease was $5.6 \pm 0.9$ years.

Non-segmental vitiligo was the most common type of the disease in 140 (90\%) reviewed patients, while segmental vitiligo appeared only in 15 (10\%) patients. The most involved area of vitiligo was the face (62\%). Of the 75 girls, 70 (93.3\%) had NSV and 5 (6.7\%) had SV. Of the 80 boys, 70 (87.5\%) had NSV; while 10 (12.5\%) had SV. There was no statistical significance between gender and clinical types of vitiligo. Family history was found in only 14 patients.

Laboratory testing was performed in all children. Two patients already diagnosed with Hashimoto's thyroiditis were female who had NSV.

One hundred and fifty-three children, with no history of thyroid dysfunction, were screened for the first time at our institute. Of these screened patients, a total of 34 from 140 with NSV had one or more thyroid parameters converted (TSH, T4, T3, anti-TPO, anti-Tg ab), whereas none of the 15 patients with segmental vitiligo had any thyroid abnormality. This was statistically significant $(p<0.05)$. Sixteen patients had thyroid abnormalities without elevated antibodies; 11 of them had elevated T3, 1 of them had a low T3 level and 4 of them had isolated elevated TSH levels.

Elevated antibodies were found in 18 patients; 11 of them had anti-TPO Ab and evidence of thyroid abnormalities: 1 subclinical hypothyroidism (> TSH), 1 subclinic hyperthyroidism $(<\mathrm{TSH})$. None of these patients reported changes in activity, palpitations, heat or cold intolerance, hyperhidrosis or any hypothyroidism symptoms. Four of them had anti-Tg Ab and evidence of thyroid abnormalities, and only 1 had isolated T4 decreased. In these 18 patients, 3 had anti TPO and anti-Tg both elevated with no thyroid function abnormality. All of these 18 patients were NSV. For gender only anti-Tg was higher in girls than in boys and this was statistically significant $(p<0.05)$.

\section{Discussion}

Vitiligo is the most common acquired depigmentation disorder and affects approximately $1 \%$ of the world's population $[9,10]$. The presence of the disease can lead to psychological trauma in these children and their parents.

Recent studies from several countries have shown that there is another association between vitiligo and thyroid diseases. However, there are limited data concerning paediatric patients. This report is from Turkey about this association in paediatric patients with vitiligo.

Firstly, our findings show that there is an association between thyroid disease and the NSV. lacovelli et al. showed that $10.7 \%$ of paediatric patients with vitiligo had thyroid abnormalities and all patients with the segmental type had normal thyroid function test results [6]. In the USA, Pagovich et al. found thyroid abnormalities in $25 \%$ of paediatric vitiligo patients and they reported that none of the patients with thyroid disease had segmental vitiligo [4]. Kroon et al. reported that $17 \%$ of children with vitiligo had thyroid abnormalities [8] and in other studies results were $11.2 \%$ and $11.8 \%$, respectively $[9,10]$. Cho et al. found a low incidence (5.9\%) but they performed only thyroid function tests [11]. Our data showed that $22 \%$ of patients had thyroid test abnormalities, $12 \%$ had autoantibody elevation and these patients were all NSV patients.

Studies showed that vitiligo/NSV involves a number of specific genes, many of which are shared with other autoimmune diseases, including autoimmune thyroid disease [12-15]. Our study results support these findings with a NSV dominance.

Data relating to gender showed that girls with vitiligo/NSV are more prone to develop autoimmune thyroid diseases than boys. In 18 patients, 14 were girls. Our results agree with other studies that showed a higher frequency of AT in females with vitiligo than in males [5-7]. Anti-TPO is documented as a sensitive tool for the determination of early subclinical ATD $[16,17]$. Studies have shown that anti-Tg ab and anti-TPO are present in Graves' disease and Hashimoto thyroiditis up to 7 years before clinical diagnosis and were elevated compared with controls. These levels increased over time before clinical diagnosis of Graves' disease but were elevated at all time points for Hashimoto's thyroiditis [18]. In this study we used anti-TPO and anti-Tg ab for detecting autoimmune thyroid disease.

Diagnosis is particularly important in children, both for the evident effect on their growth and because early onset of the disease is associated with a bad prognosis and also has a predisposition to become chronic [19].

\section{Conclusions}

We recommend that, for screening children with non-segmental vitiligo for thyroid disease, measuring anti-TPO, anti-Tg and thyroid function tests could be useful.

\section{Conflict of interest}

The authors declare no conflict of interest.

\section{References}

1. Cui J, Bystryn JC. Melanoma and vitiligo are associated with antibody responses to similar antigenson pigment cells. Arch Dermatol 1995; 131: 314-8. 
2. Ogg GS, Rod Dunbar P, Romero P, et al. High frequency of skin-homing melanocyte-specific cytotoxic T lymphocytes in autoimmune vitiligo. J Exp Med 1998; 188: 1203-8.

3. Ezzedine K, Diallo A, Léauté-Labrèze C, et al. Multivariate analysis of factors associated with early-onset segmental and nonsegmental vitiligo: a prospective observational study of 213 patients. Br J Dermatol 2011; 165: 44-9.

4. Pagovich OE, Silverberg JI, Freilich E, Silverberg NB. Thyroid abnormalities in pediatric patients with vitiligo in New York City. Cutis 2008; 81: 463-6.

5. Gey A, Diallo A, Seneschal J, et al. Autoimmune thyroid disease in vitiligo: multivariate analysis indicates intricate pathomechanisms. Br J Dermatol 2013; 168: 756-61.

6. lacovelli P, Sinagra JL, Vidolin AP, et al. Relevance of thyroiditis and of other autoimmune diseases in children with vitiligo. Dermatology 2005; 210: 26-30.

7. Uncu S, Yaylı S, Bahadır S, et al. Relevance of autoimmune thyroiditis in children and adolescents with vitiligo. Int J Dermatol 2011; 50: 175-9.

8. Kroon MW, Vrijman C, Chandeck C, et al. High prevalence of autoimmune thyroiditis in children and adolescents with vitiligo. Horm Res Paediatr 2013; 79: 137-44.

9. Prćić S, Djuran V, Katanić D, et al. Vitiligo and thyroid dysfunction in children and adolescents. Acta Dermatovenerol Croat 2011; 19: 248-54.

10. Yang $\mathrm{Y}$, Lin X, Fu W, et al. An approach to the correlation between vitiligo and autoimmune thyroiditis in Chinese children. Clin Exp Dermatol 2010; 35: 706-10.

11. Cho SB, Kim JH, Cho S, et al. Vitiligo in children and adolescents: association with thyroid dysfunction. J Eur Acad Dermatol Venereol 2011; 25: 64-7.

12. Lerner AB. Vitiligo. J Invest Dermatol 1959; 32: 285-310.

13. Alikhan A, Felsten LM, Daly M, Petronic-Rosic V. Vitiligo: a comprehensive overview. Part I. Introduction, epidemiology, quality of life, diagnosis, differential diagnosis, associations, histopathology, etiology, and work-up. J Am Acad Dermatol 2011; 65: 473-91.

14. Spritz RA. Shared genetic relationships underlying generalized vitiligo and autoimmune thyroid disease. Thyroid 2010; 20: 745-54.

15. Alkhateeb A, Fain PR, Thody A, et al. Epidemiology of vitiligo and associated autoimmune diseases in Caucasian probands and their families. Pigment Cell Res 2003; 16: 208-14.

16. Kemp EH. Autoantibodies as diagnostic and predictive markers of vitiligo. Autoimmunity 2004; 37: 287-90.

17. Doullay F, Ruf J, Codaccioni JL, Carayon P. Prevalence of autoantibodies to thyroperoxidase in patients with various thyroid and autoimmune diseases Autoimmunity 1991; 9: 237-44.

18. Hutfless S, Matos P, Talor MV. Significance of prediagnostic thyroid antibodies in women with autoimmune thyroid disease. J Clin Endocrinol Metab 2011; 96: E1466-71.

19. Halder RM. Childhood vitiligo. Clin Dermatol 1997; 15: 899906. 